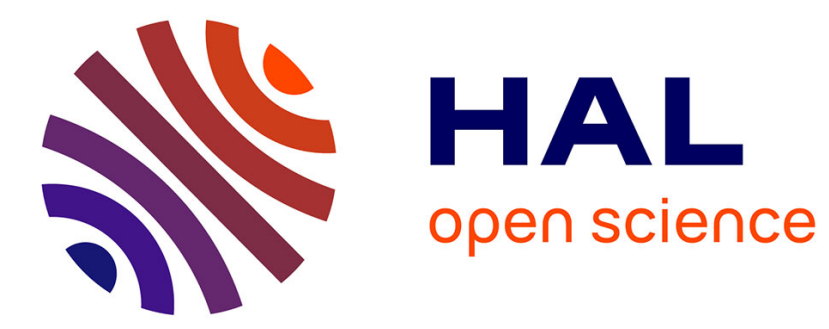

\title{
Combining low and high electron energy diffractions as a powerful tool for studying $2 \mathrm{D}$ materials
}

\author{
Pascal Puech, Iann C Gerber, Fabrice Piazza, Marc Monthioux
}

\section{To cite this version:}

Pascal Puech, Iann C Gerber, Fabrice Piazza, Marc Monthioux. Combining low and high electron energy diffractions as a powerful tool for studying 2D materials. Applied physics. A, Materials science \& processing, 2021, 127, pp.485. 10.1007/s00339-021-04568-9 . hal-03431279

\section{HAL Id: hal-03431279 \\ https://hal.science/hal-03431279}

Submitted on 16 Nov 2021

HAL is a multi-disciplinary open access archive for the deposit and dissemination of scientific research documents, whether they are published or not. The documents may come from teaching and research institutions in France or abroad, or from public or private research centers.
L'archive ouverte pluridisciplinaire HAL, est destinée au dépôt et à la diffusion de documents scientifiques de niveau recherche, publiés ou non, émanant des établissements d'enseignement et de recherche français ou étrangers, des laboratoires publics ou privés. 


\title{
Combining Low and High Electron Energy Diffractions as a Powerful Tool for Studying 2D Materials
}

\author{
Pascal Puech ${ }^{1 *}$, Iann C. Gerber ${ }^{2}$, Fabrice Piazza ${ }^{3}$, Marc Monthioux ${ }^{1 *}$
}

${ }^{1}$ Centre d'Elaboration des Matériaux et d'Etudes Structurales (CEMES), UPR-8011 CNRS, Université de Toulouse, France.

${ }^{2}$ Laboratoire de Physico-Chimie des Nano-Objets (LPCNO), UMR 5215 CNRS, INSA, Université de Toulouse, France.

${ }^{3}$ Laboratorio de Nanociencia, Pontificia Universidad Católica Madre y Maestra, Santiago de Los Caballeros, Dominican Republic.

*Corresponding authors: pascal.puech@cemes.fr; marc.monthioux@cemes.fr

\begin{abstract}
Two-dimensional (2D) materials are among the most studied ones nowadays, because of their unique properties. They are made of single- or few-atom-thick layers whose variation in the stacking sequence may result in a variety of crystallographic structures, whether they are assembled by van der Waals forces or covalently bonded. Although identifying both the number of layers and the stacking sequence is of an utmost importance because of the driving role these parameters have on the properties, there is currently no technique available to do so. We demonstrate here that combining low energy (1-10 keV) electron diffraction with the usual high energy $(>50 \mathrm{keV})$ electron diffraction on the same 2D object is able to fill the gap. We illustrate this by taking the examples of a variety of 2D materials, built from either a single-type of atom with low $Z$-number such a graphene $(\mathrm{C})$, or two types of atoms with low $Z$ number such as diamane $\left(\mathrm{C}_{2} \mathrm{H}\right)$, or two types of atoms with high $Z$-number such as $\mathrm{MoS}_{2}$. Meanwhile, we propose a simplified method for comparing calculated patterns to experimental ones, and discuss the limitation of the technique.
\end{abstract}

Keywords: 2D materials, electron diffraction, modelling, stacking identification, graphene, diamane

\section{Declarations}


Funding: This work is funded by the EUR grant NanoX $n^{\circ}$ ANR-17-EURE-0009 in the framework of the French "Programme des Investissements d'Avenir" and by the Ministry of Higher Education, Science and Technology of the Dominican Republic (2016-2017 and 2018 FONDOCyT programs)

Conflict of Interest: Authors declare no conflict of interest.

Availability of data and material: Most of the data are provided either in the main article, or a Supplementary Information. Any data missing or materials are available upon request to the corresponding author.

Code availability: Code can be made available upon request to the corresponding author.

Authors' contributions: P.P. is at the origin of the methodology principle and performed the calculations. I.G. contributed to the calculations and supplied information on $\mathrm{MoS}_{2}$. F.P. provided the 2LG materials and carried-out the low-voltage electron diffraction work. M.M. wrote most of the paper. All the authors contributed to discussing the results and the manuscript versions.

\section{Introduction}

Transmission electron microscopy (TEM) is often used for characterizing 2D layers. While the direct imaging is often not able to determine the stacking sequence, electron diffraction may have this ability. Taking the example of few-layer graphene (FLG), it is of an utmost importance to identify both the number of layers and the stacking sequence, because of the driving role these parameters have on the properties. For this purpose, analyzing the relative spot intensities of electron diffraction patterns (DPs) using a single wavelength selected within the 60-300 keV energy range is commonly used [110] along with attempts to vary the number of layers [1,5,7,8] and the specimen tilt angle [2-4]. For instance, the determination of the stacking sequence in FLG was proposed by Ping and Fuhrer [7] to be achieved by analyzing the relative spot intensities in electron diffraction patterns, however requiring that the FLG to be analyzed exhibit incremental layer steps. As only one type of atoms is present, the relative intensities are not affected by the imaginary part of the atomic form factor because of its factorization. Moreover, multiple third-order scattering process, as proposed by Deb et al. [11] has no role here due to the absence of polarity in the primitive cell. Also, converting graphene films into other materials upon post-treatments is a synthesis route which is developing [10,12-16]. For $\mathrm{MoS}_{2}$, considering the imaginary part of the atomic form factor splits the 6-fold symmetry in two 3-fold 
patterns, alternating strong and weak spots [17]. Although the relationship between weak peaks and atomic orientation is well understood, relying on the absolute intensity values is not straightforward because of others effects. For instance, multiple scattering events within thick, non-centrosymmetric crystals were recently proposed to be considered for estimating more accurately the scattered intensities [11].

Nowadays, multiwavelength electron diffraction analysis has become possible, thanks to the availability on market of TEM equipment able to operate between 5 and $300 \mathrm{kV}$. Therefore, it is of primary importance to know the differences between DPs obtained while varying electron wavelength. With pure carbon materials, an acceleration voltage of $80 \mathrm{kV}$ is the maximum possible for reliable TEM observations due to the kinetic energy transfer from the incoming electrons to the atom nuclei (knock-on damage) [18]. As the $\mathrm{H}$ atoms involved in $\mathrm{C}-\mathrm{H}$ bonds exhibit a mass difference of $\sim 12$, operating at $5 \mathrm{kV}$ should ideally be the preferred choice for observing materials such as graphane and diamane as carried-out by Piazza et al. [19] for not inducing any irradiation damage. Thus, comparing materials to each other, or comparing a single material to itself after transformation, by investigating them at several electron wavelengths can be done although it requires some cautions. Consequently, in this paper, we discuss extensively the distribution of spot intensities in DPs while varying the electron energy in the 1-100 keV range for a selection of 2D materials. Meanwhile, the great benefit of performing multi-wavelength electron diffraction in the identification of stacking sequences in $2 \mathrm{D}$ materials is revealed.

\section{Theory}

2D materials exhibit the specificity of having the reciprocal spots corresponding to the material periodicities to be elongated perpendicularly to the thinned direction, and modulated according to the number of stacked layers (see Section 1 in Supplementary Information (SI)). In order to investigate the influence of varying the electron energy on the scattered intensities, we have simulated a selection of electron diffraction patterns (DPs) first considering graphene systems made of a variety of layer number $N$ under various stacking configurations, such as $\mathrm{A}, \mathrm{AA}, \mathrm{AB}, \mathrm{ABA}$ or $\mathrm{ABC}$, while using an electron energy ranging from 100 to $1 \mathrm{keV}$. The relative positions of layers $\mathrm{A}, \mathrm{B}$, and $\mathrm{C}$ in the cell are reminded in SI (Section 2).

DPs were obtained thanks to innovative calculation principles as follows (also see SI, Section 3): 


$$
S_{\text {electron }}\left(\vec{q}, \theta_{\vec{q}}\right)=B \frac{1}{N}\left|\sum_{e l_{i}=1}^{N_{e l} \text { in the primitive cell }} f_{e l_{i}}(q) \sum_{i \in e l_{i}} \exp \left(i\left[2 \pi\left(h x_{i}+k y_{i}\right)+q_{z} z_{i} c\right]\right)\right|^{2}
$$

where $x_{i}, y_{i}$ and $z_{i}$ are the relative coordinates in the cell. Taking graphene as an example, it corresponds to 2 atoms in each plane when all planes are stacked in the $z$ direction, where $h$ and $l$ are the Laue indices. $q_{z}$ is the vector in the reciprocal space involved in the diffraction process (see $\mathbf{S I}$ ). With simple algebra, we obtain:

$$
q_{z}=\frac{2 \pi}{\lambda}-\sqrt{\left(\frac{2 \pi}{\lambda}\right)^{2}-G^{2}}
$$

where $G$ is a vector of the reciprocal space in the $\left(k_{x} k_{y}\right)$ plane.

For materials composed of only one type of atoms, the atomic form factor $f_{e l_{i}}(q)$ can be factorized and its imaginary part does not change the phase of the summation in Eq.1. When several types of atoms form the primitive cell, the imaginary part of the atomic form factor acts differently: for carbon atom, the $\mathrm{K}$ absorption edge (the highest energy one) is around $280 \mathrm{eV}$ only while the $f(q)$ module is changed by its imaginary part by less than $5 \times 10^{-6}$. Consequently, for more energetic electron wavelengths such as those from most of studies including the present one, the imaginary part effect is negligible. Therefore, as long as low Z-number atoms are involved, such as for FLG, hydrogenated carbon structures (i.e., graphane, diamane...), or BN for instance, the imaginary part of atomic form factor plays nearly no role. Shevitski et al. [8] found $0.5 \%$ of scattering per graphene layer, supporting the fact that for few layers of graphene, multiple scattering can be neglected.

In our approach, considering the in-plane extension to be infinite imposes on the reciprocal vector $\mathrm{G}$ to be in the plane. Along the $z$ direction, the summation no longer involves a large number of cells as in regular calculations for 3D materials, but involves only few atoms. This approach is valuable thanks to its simplicity and rapidity.

The reliability and relevance of the calculation principles described above were validated by comparing with the results obtained by a regular DP calculation software [20] (see SI, Section 4). Identical results were obtained.

For materials involving more than a single type of atom and assuming an absorption edge in the range of 1 to $100 \mathrm{keV}$ (starting from $\mathrm{Na}$ with $Z=11$ ), anomalous scattering has to be considered, leading to the additional contribution of the imaginary part. Thus, for 2D materials made of several different atoms as in transition metal dichalcogenides (TMDs), such as $\mathrm{MoS}_{2}$, the imaginary part of the atomic form factor can play a significant role and lift the symmetry (e.g., the 6-fold axis may become a 
3-fold axis). Moreover, other effects can be present, such as multiple scattering events. Both effects will be discussed in detail in the following.

Our calculation principle is simple and uses measured $f(q)$ values. The use of $q_{z}$ as defined by equation (2) has been suggested [8] but never incorporated in the calculation in such a way revealing the differences induced in the electron diffraction patterns by changing the energy of the incoming electrons regarding the distribution of the scattered intensities.

\section{Methods}

Suspended bi-layer (2LG) graphene films grown by chemical vapor deposition and deposited onto 3 mm-diameter gold Quantifoil TEM grids were supplied from Graphenea (https://www.graphenea.com). The films are polycrystalline and were obtained from the successive transfer of two individual single-layer graphene. The resulting 2LG stacking sequence type therefore varies randomly across the film, with crystallite sizes not larger than $20 \mu \mathrm{m}$.

Diamane was prepared by a low-temperature, pressureless hydrogenation process of the 2LG material above-mentioned according to the conditions reported in Piazza et al. [16].

Electron diffraction was carried-out using a low voltage $(5 \mathrm{kV})$ table top transmission electron microscope equipped with a Schottky electron source from Delong Instruments. Electron diffraction patterns were obtained in selected area mode from $100 \mathrm{~nm}$-large areas.

The principles and basic equations for the calculations are provided in the Supporting Information.

\section{Application to 1- to 4-layer graphenes}

2D graphene materials exhibit DPs with two main rings, which will be named Ringl (inner ring) and Ring2 (outer ring) respectively, both usually wearing 6 spots distributed according to a 6-fold symmetry (examples are plenty in the literature, and some will be provided later on). Examples of how the intensity features in the calculated DPs vary for the two rings, Ring1 and Ring2, for a selection of different stacking sequences involving up to 3 layers, when varying the electron energy continuously from 1 to $100 \mathrm{keV}$ are provided in Figure 1.

Considering plots such as in Figure 1 (and more reported up to 6 layers in Table S4 in SI) suggests that comparing spot intensities and intensity ratios should allow building an identification chart based 
on varying the wavelength. As the results in the $60-100 \mathrm{keV}$ range do not differ significantly (Figure 1), we propose as Table 1 such an identification chart by merely using 5 and $100 \mathrm{keV}$, respectively. Table 1 reports all the calculated intensity values for seven different stacking sequences. Interestingly, a given stacking sequence which does not exhibit any singularity at high electron energy value, making difficult its identification, can exhibit one at low electron energy value, or the other way. Therefore, identifying each of the stacking sequences, which was difficult or impossible so far when only using the standard $60-100 \mathrm{keV}$ electron energies, now appears to be possible and easy by combining DPs at low and high electron energies when needed.

In Table 1 are reported in bold the data which are believed to be characteristic of the stacking configurations considered. For some cases, e.g., bilayer graphene with AB stacking (2LG-AB), a single wavelength is enough (here for $5 \mathrm{keV}$ ), as it is the only case for which the three-fold symmetry appears strongly on Ringl, while the intensity difference between Ringl (considering the strong spots) and Ring2 is low. It is worth noting, however, that the calculated Ring2/Ring1 intensity ratio values of 0.9 and 3 reported here for $1 \mathrm{LG}$ and $2 \mathrm{LG}-\mathrm{AB}$, respectively, at $100 \mathrm{keV}$, is fairly consistent with both the experimental and calculated values of $\sim 0.9$ and $\sim 3.5$, respectively, obtained from Meyer et al. (at $60 \mathrm{keV}$ ) [2], although the calculation method was different (Fourier Transform of the projected atomic potentials).

In order to support what is claimed above and in the SI, examples of experimental DPs are shown and compared to calculated ones. On top of Figure 2 are provided calculated DPs obtained at $5 \mathrm{keV}$ electron energy for the first three stacking configurations of Table 1 along with the corresponding experimental DPs at the bottom of the Figure, also obtained at $5 \mathrm{keV}$.

The diffracted spot intensity features at $5 \mathrm{keV}$ electron energy for single graphene (A, Figure 2a) in both the calculated and experimental DPs appear similar to what is reported in the literature for regular high energy (e.g., $>50 \mathrm{keV}$ ) DPs $[1,2,22,23]$. On the other hand, the intensity difference between Ring1 and Ring2 for 2LG-AA is enough to be discernible to eye in both the calculated and experimental DPs (Figure 2b), which could not be reported for high energy DPs of graphene in the literature, and has never been. Likewise, for 2LG-AB, the variation of the spot intensities along Ring 1 giving rise to a three-fold symmetry instead of the expected 6-fold one appears clearly (Figure 2c), whereas this intensity variation is too faint at high electron energy for having been observable and reported in the literature. The latter observations are unprecedented, they are fully consistent with what is reported in Figure 1 and Table 1, and therefore strongly support our claim. 


\section{Application to graphane and diamanes}

As another example of 2D materials, however built from more than a single type of atom, diamanes are now considered. Diamanes are obtained from the $s p^{2}$-to-s $s p^{3}$ conversion of 2LG graphene promoted by a hydrogenation reaction on the basal plane on both faces $[12,19]$.

With full hydrogenation, the graphene lattice parameter expands from 2.46 to $\sim 2.53 \AA$. However, this parameter varies locally with the extent of hydrogenation, hence only using it to discriminate between the various forms of diamane, or graphane from diamanes, is not accurate, except to state that some hydrogenation event has happened. Surprisingly, Elias et al. [13], who claimed for the synthesis of graphane, observed a contraction of the lattice parameter from analyzing the DPs. This actually shows a deviation to perfect crystal and that genuine graphane was in fact not formed, and/or very likely modified due to the high electron energy used $(300 \mathrm{keV})$. Indeed, graphane and diamanes are quite sensitive to electron irradiation for such a high energy (and even at lower energies, e.g., $60 \mathrm{keV}$ ), because of the light weight of $\mathrm{H}$ atoms and their resulting sensitivity to knock-on events promoted by the electron beam [14]. This prevents any reliable comparison between DPs obtained at low and safe electron energy (e.g., $5 \mathrm{keV}$ ) and at higher energies to be made. For such materials, DPs should be taken at very low electron energy. As a matter of fact, for fluorinated diamane, because $\mathrm{F}$ is heavier than $\mathrm{C}$, hence than $\mathrm{H}$, an electron energy of $80 \mathrm{keV}$ was stated to be enough for maintaining the material stability and obtaining reliable DPs [10].

Because graphane and diamanes have a cubic or hexagonal structure with either the [111] or [001] axes perpendicular to the material foil plane, their DPs are expected to also exhibit the same two rings

with 6-fold symmetry as FLG crystals. As the material contains two types of atoms, C and H, the atomic form factor cannot be factorized. However, as both atoms have a low $Z$ number, the imaginary part plays a negligible role (see Table 2 with Ringl differences less than $0.2 \%$ evidenced for diamaneAA). The same calculations as for FLGs were then made for graphane and diamanes, whose results are reported in Table 2:

For graphane, it is interesting to see that, while no intensity variation could even be seen experimentally in $\operatorname{Ring} 1\left(\operatorname{Ring} l_{(\mathrm{s})} / \operatorname{Ring} l_{(\mathrm{w})}=1.1\right)$ at $100 \mathrm{keV}$, the intensity ratio of the strong over weak spots is fortunately amplified up to 1.4 at $5 \mathrm{keV}$, i.e. at the estimated limit of the measurement reliability. This gives hope that the intensity variation could actually be seen and discriminated from 
diamane-AA at $5 \mathrm{keV}$. On the other hand, diamane-AA discriminates fairly well from Diamane-AB at $5 \mathrm{keV}$, thanks to both their $\operatorname{Ring} l_{(\mathrm{s})} / \operatorname{Ring} l_{(\mathrm{w})}$ and $\operatorname{Ring} 2 / \operatorname{Ring} l_{(\mathrm{s})}$ ratios.

\section{Application to TMDs $\left(\mathrm{MoS}_{2}\right)$}

We have also applied our methodology to $\mathrm{MoS}_{2}$, as an example of TMDs, i.e., 2D materials built from two types of atoms, both with high $Z$ numbers. TMDs are also materials resulting from the assembly of layers by van der Waals forces, as FLGs. Because each layer includes 3 sub-layers (1 Mo layer between $2 \mathrm{~S}$ layers) within which the relative spatial display of the atoms may vary, there are more structure variations than in graphene, and the overall atomic form factor is more complicate than for pure carbon material. In addition, due to the higher atomic numbers of the atoms involved, the energy of the incident wave in the 1-100 keV range can overlap some of the absorption edges of Mo and $\mathrm{S}$ (see Figure S5 in SI), leading to a substantial imaginary part of the atomic form factor. Moreover, as for hydrogenated carbon, it is not possible to factorize the atomic form factor; therefore, the imaginary part that we have estimated (see SI) contributes significantly to the absolute value of the scattered intensity. A complex atomic factor introduces a phase, and this phase modifies the exponential term, leading to lifting the degeneracy between 10 and $\overline{1} 0$ indices for example. The corrections related to the inelastic scattering can be easily incorporated with our methodology and we have included them as explained in the SI. The absorption edges are clearly visible in Figure 3a. Due to the existence of the two Mo and S sublattices, the lattice symmetry is reduced from 6-fold to 3-fold which can be used to obtain the crystallite orientation [17]. Because of both the variety of cases and the several effects which have to be considered, we will not provide the same detailed analysis as for the graphene system above. To use the data from the literature, we focus our attention to the $1 H$-structure of monolayer $\mathrm{MoS}_{2}$. Other cases (1T, and several layers) are reported in SI. In Figure 3, our calculation shows anomalies due to the imaginary part of the atomic form factors, which are seen to perturbate the intensity versus energy curves at $\sim 2.5$ and $20 \mathrm{keV}$. They correspond to the energy values of the $\mathrm{K}$ and $\mathrm{L}$ adsorption edges for both $\mathrm{S}$ and Mo (see Figure S5 in SI). Also, multiple scattering events play a role at high incident electron energy in the case of $\mathrm{MoS}_{2}$, hence they have to be considered [11]. Thus, we have plotted the Ringl(s) $/$ Ring $1_{(\mathrm{w})}$ intensity ratio in Figure $3 \mathrm{~b}$. Both the imaginary part of the atomic form factor (blue curve) and multiple-scattering effects (magenta curve, see Figure $3 b$ ) prevent our method to be used in the same univocal and easy way as for graphene and diamanes. 
Thus, using our methodology for TMDs is possible provided a full calculation specific to each TMD and including the imaginary part and the multiple scattering effects is carried-out. This is beyond the scope of this study.

\section{Conclusions}

We showed how the relative intensities between the various spots in diffraction patterns of $2 \mathrm{D}$ materials made of a single type of atom can be easily obtained for a large range of incident electron wavelengths (energies) by factorizing the imaginary part of the atomic form factor. The study revealed major discrepancies between the intensity distributions obtained at low (e.g., 1-10 keV) and high (e.g., $>50 \mathrm{keV}$ ) energies. This suggested that combining both could be an efficient technique to identify stacking sequences in few-layer graphenes (C), which considering a single high energy electron source cannot do. Our methodology was demonstrated to be also valid for more complex 2D materials such as diamanes $\left(\mathrm{C}_{2} \mathrm{H}\right)$, and is predicted the same for other 2D materials made of atoms with low $Z$ number (below $\mathrm{Na}$ ) such as $\mathrm{h}-\mathrm{BN}$, because the imaginary part of the related atomic form factors can be neglected. Our approach remains simple and easy as long as the multiple-scattering effects also remain negligible. On the other hand, although the principle remains valid for 2D materials made of atoms with high Z-numbers such as transition metal dichalcogenides (illustrated by $\mathrm{MoS}_{2}$ ), the simplified calculation method proposed here cannot be used, and attention has to be paid not to overlap the absorption edges of the atoms involved.

More generally speaking, the novelty and input of our work regarding the study of 2D materials (hence with a limited number of layers so that to minimize the multiple scattering events) is then quadruple: $(i)$ it reveals the differences induced in the electron diffraction patterns regarding the distribution of the scattered intensities when changing the energy of the incoming electrons. Among other implications, this means that comparing results from different papers which use different electron energies should be made carefully; (ii) it introduces a simplified method to obtain calculated electron diffraction patterns; (iii) it proposes to consider combining both low and high electron energy diffractions as a new technique to study 2D materials; (iv) it reveals that, should a single electron energy be used (because of time constraints, equipment availability, or material sensitivity to irradiation) low energy (1-10 keV) is preferable as more able to reveal characteristic features in in the diffracted spot intensity variation. 
Supporting Information are provided, reminding the basics on the possible stacking sequences in graphene, giving details on the methodology proposed to calculate spot intensities in the diffraction patterns, and providing further data.

\section{Acknowledgements}

The authors thank R. Ruoff for stimulating discussions.

\section{References}

1. S. Horiuchi, T. Gotou, M. Fujiwara, R. Sotoaka, M. Hirata, K. Kimoto, T. Asaka, T. Yokosawa, Y. Matsui, K. Watanabe, M. Sekita, Jpn. J. Appl. Phys. 42, L1073 (2003)

2. J. C. Meyer, A. K. Geim, M. I. Katsnelson, K. S. Novoselov, T. J. Booth, S. Roth, Nature. 446, 60 (2007)

3. J. C. Meyer, A. K. Geim, M. I. Katsnelson, K. S. Novoselov, D. Obergfell, S. Roth, C. Girit, A. Zettl, Sol. State Comm. 143, 101 (2007)

4. Y. Hernandez, V. Nicolosi, M. Lotya, F. M. Blighe, Z. Sun, S. De, I. T. McGovern, B. Holland, M. Byrne, Y. Gun'ko, J. Boland, P. Niraj, G. Duesberg, S. Krishnamurti, R. Goodhue, J. Hutchison, V. Scardaci, A. C. Ferrari, J. N. Coleman, Nature Nanotechnol. 3, 563 (2008)

5. Y. J. Suh, S. Y. Park, M. J. Kim, Microsc. Microanal. 15, 1168 (2009)

6. N. Behabtu, J. R. Lomeda, M. J. Green, A. L. Higginbotham, A. Sinitskii, D. V. Kosynkin, D. Tsentalovich, A. N. G. Parra-Vasquez, J. Schmidt, E. Kesselman, Y. Cohen, Y. Talmon, J. M. Tour, M. Pasquali, Nature Nanotechnol. 5, 406 (2010)

7. J. Ping, M. S. Fuhrer, Nano Lett. 12, 4635 (2012)

8. B. Shevitski, M. Mecklenburg, W. A. Hubbard, E. R. White, B. Dawson, M. S. Lodge, M. Ishigami, B. C. Regan, Phys. Rev. B. 87, 045417 (2013)

9. T. Latychevskaia, S.-K. Son, Y. Yang, D. Chancellor, M. Brown, S. Ozdemir, I. Madan, G. Berruto, F. Carbone, A. Mishchenko, K. S. Novoselov, Front. Phys. 14, 13608 (2019)

10. P. V. Bakharev, M. Huang, M. Saxena, S. W. Lee, S. H. Joo, S. O. Park, J. Dong, D. C. Camacho-Mojica, S. Jin, Y. Kwon, M. Biswal, F. Ding, S. K. Kwak, Z. Lee, R. S. Ruoff, Nature Nanotechnol. 15, 59 (2020)

11. P. Deb, M. C. Cao, Y. Han, M. E. Holtz, S. Xie, J. Park, R. Hovdena, D. A. Muller, Ultramicrosc. 215, $113019(2020)$

12. L. A. Chernozatonskii, P. B. Sorokin, A. Kvashnin D. G. Kvashnin, J. Exp. Theor. Phys. Lett. 90, 134 (2009)

13. D. C. Elias, R. R. Nair, M. G. Mohiuddin, S. V. Morozov, P. Blake, M. P. Halsall, A. C. Ferrari, D. W. Boukhvalov, M. I. Katsnelson, A. K. Geim, K. S. Novoselov, Science. 323, 610 (2009)

14. F. Piazza, K. Gough, M. Monthioux, P. Puech, I. Gerber, R. Wiens, G. Paredes, C. Ozoria, Carbon. 145, 10 (2019)

15. F. Piazza, M. Monthioux, P. Puech, I. Gerber, Carbon. 156, 234 (2020)

16. F. Piazza, K. Cruz, M. Monthioux, P. Puech, I. Gerber, Carbon. 169, 129 (2020)

17. Z. Wang, S. Ning, T. Fujita, A. Hirata, M. Chen, ACS nano. 10, 10308 (2016)

18. B. E. Smith, D. E. Luzzi, J. Appl. Phys. 90, 3509 (2001)

19. F. Piazza, M. Monthioux, P. Puech, I. C. Gerber, K. Gough, J. Carbon Res. 7, 9 (2021) 
20. V. S. Neverov, SoftwareX. 6, 63 (2017)

21. D. Sen, K. S. Novoselov, P. M. Reis, M. J. Buelher, Small. 6, 1108 (2010)

22. A. Dato, V. Radmilovic, Z. Lee, J. Phillips, M. Frenklach, Nano Lett. 8, 2012 (2008)

23. K. Kim, Z. Lee, W. Regan, C. Kisielowski, M. F. Crommie, A. Zettl, ACS nano. 5, 2142 (2011) 

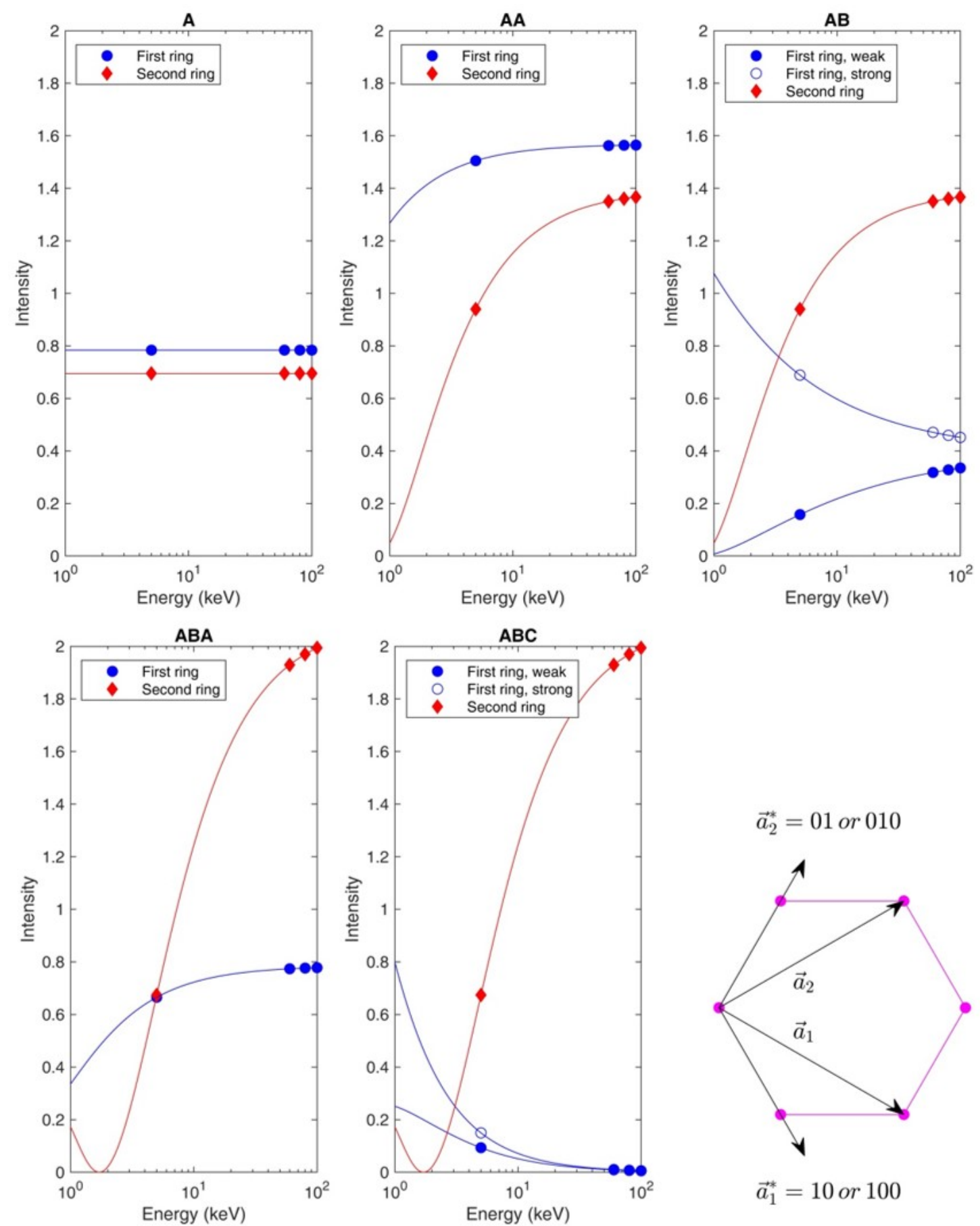

$\vec{a}_{1}^{*}=10$ or 100

Fig. 1 Calculated variation of the diffracted spot intensities while continuously varying the electron energy from 1 to $100 \mathrm{keV}$, and for various stacking sequence types in graphene. Intensities are that for a single spot from each ring, Ringl (blue line and circles) and Ring2 (red line and diamonds). When a three-fold symmetry of the intensity distribution appears on Ring1, two intensity values are provided corresponding to that of one of the weak spots (solid circle) and one of the strong spots (open circle), respectively. Intensities specifically obtained for electron energies of 5, 60, 80, and $100 \mathrm{kV}$ TEM acceleration voltage are marked, for easier recognition. For $\mathrm{AB}$ and $\mathrm{ABC}$ stacking, the weak intensity corresponds to 10 or 100 Laue indices, which can be used to determine the FLG in-plane orientation 


\begin{tabular}{|l|c|c|c|c|c|c|c|c|c|c|}
\hline \multirow{2}{*}{$\begin{array}{l}\text { Stacking } \\
\text { sequence }\end{array}$} & \multicolumn{5}{|c|}{$\mathbf{5 ~ k e V}$} & \multicolumn{5}{c|}{$\mathbf{1 0 0}$ keV } \\
\cline { 2 - 10 } & $\begin{array}{l}\text { Ring 1 } \\
\text { weak }\end{array}$ & $\begin{array}{l}\text { Ring1 } \\
\text { strong }\end{array}$ & $\begin{array}{l}\text { Ring1(s)/ } \\
\text { Ring1(w) }\end{array}$ & Ring2 & $\begin{array}{c}\text { Ring2/ } \\
\text { Ring1(s) }\end{array}$ & $\begin{array}{l}\text { Ring1 } \\
\text { weak }\end{array}$ & $\begin{array}{c}\text { Ring1 } \\
\text { strong }\end{array}$ & $\begin{array}{c}\text { Ring1(s)/ } \\
\text { Ring1(w) }\end{array}$ & Ring2 & $\begin{array}{c}\text { Ring2/ } \\
\text { Ring1(s) }\end{array}$ \\
\hline A & \multicolumn{2}{|c|}{0.784} & 1 & 0.695 & $\mathbf{0 . 9}$ & \multicolumn{2}{|c|}{0.784} & 1 & 0.695 & $\mathbf{0 . 9}$ \\
\hline AA & \multicolumn{2}{|c|}{1.505} & $\mathbf{1}$ & 0.940 & $\mathbf{0 . 6}$ & \multicolumn{2}{|c|}{1.564} & 1 & 1.367 & 0.9 \\
\hline AB & $0.157^{*}$ & 0.689 & $\mathbf{4 . 4}$ & 0.940 & $\mathbf{1 . 4}$ & $0.335^{*}$ & 0.451 & 1.3 & 1.367 & 3 \\
\hline ABA & \multicolumn{2}{|c|}{0.665} & $\mathbf{1}$ & 0.674 & $\mathbf{1}$ & 0.778 & 1 & 1.994 & $\mathbf{2 . 6}$ \\
\hline ABC & $\mathbf{0 . 0 9 3}$ & 0.150 & 1.7 & 0.674 & $\mathbf{4 . 5}$ & $\mathbf{0 . 0 0 5}$ & $\mathbf{0 . 0 0 6}$ & $\sim 1$ & 1.994 & 332 \\
\hline ABAB & $\mathbf{0 . 2 6 7}$ & 1.166 & $\mathbf{4 . 4}$ & $\mathbf{0 . 2 3 4}$ & $\mathbf{0 . 2}$ & $0.665^{*}$ & 0.896 & 1.3 & 2.556 & $\mathbf{2 . 9}$ \\
\hline ABCA & $\mathbf{0 . 0 2 0}$ & $0.201^{*}$ & $\mathbf{1 0}$ & 0.234 & 1.2 & 0.162 & $0.220^{*}$ & 1.4 & 2.556 & $\sim 12$ \\
\hline
\end{tabular}

$*$ is along the $10(0)$ direction.

Table 1 Identification chart for a series of possible stacking sequences in graphene up to 4 layers (additional cases up to 6 layers in Table S4 in SI) for DPs obtained for electron energies of 5 and 100 $\mathrm{keV}$ respectively. Data in bold are assumed to be characteristic of the stacking sequence considered. Intensities reported are that of a single spot for each spot series (Ring1(weak), Ring1(strong), Ring2). It is considered that an intensity ratio in the range $\sim 0.7-1.4$ cannot provide an intensity difference high enough for being visible to eye, and can be misled by intensity variations due to local deformations (so-called corrugations [2,3]), although such corrugations rapidly become unlikely as the number of stacked graphene increases because of the exponential increase of the resulting bending modulus [21]. Detailed comments are provided in SI (Section 4) 

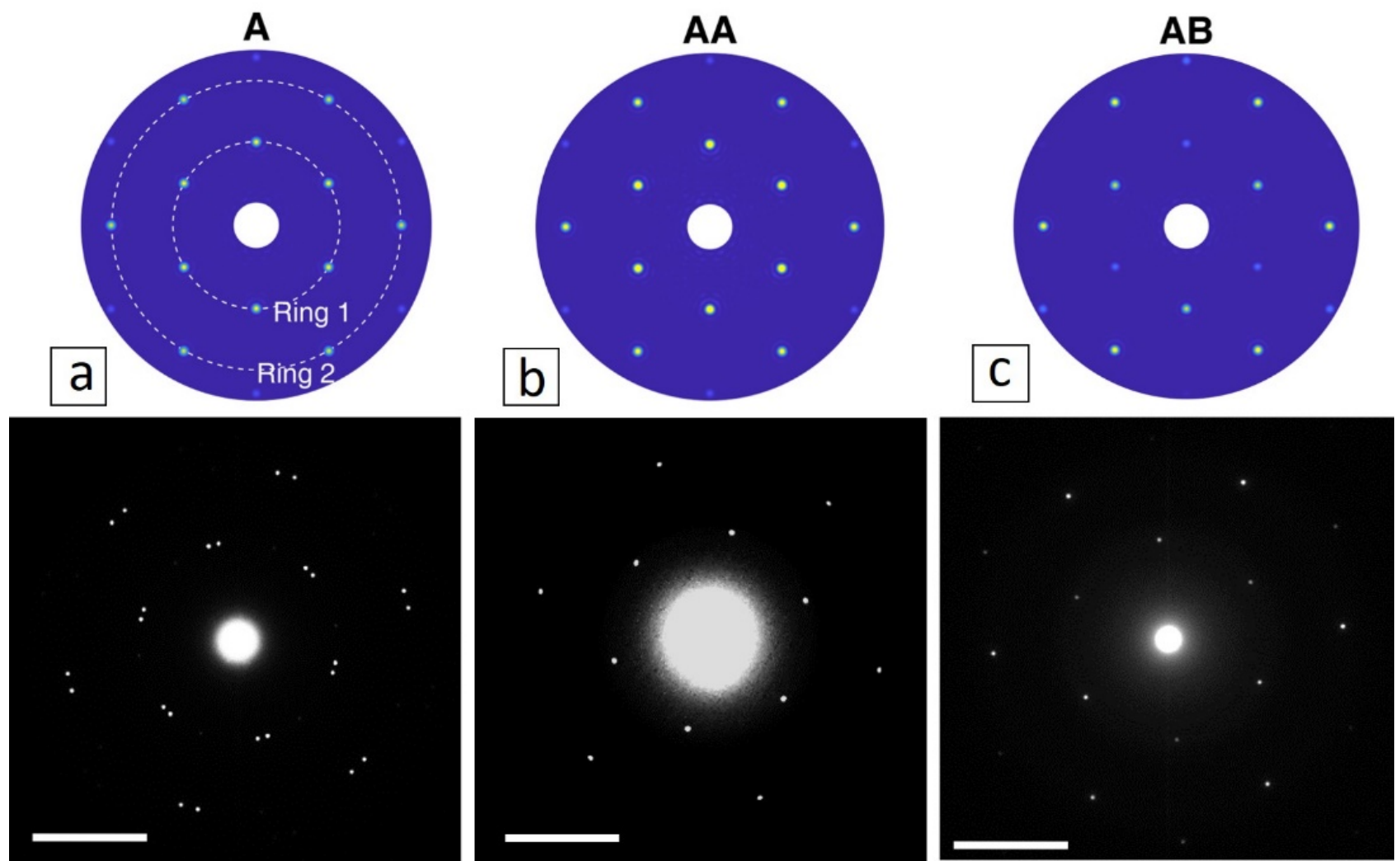

Fig. 2 Examples, for a $5 \mathrm{keV}$ electron energy, of calculated (above, using XaNSoNS, corrected by considering the electron cloud) and experimental (below, on 2LG materials) electron DPs. (a) Single graphene, where all spot intensities on Ring1 are equal and also equal to that on Ring2; the experimental DP (bottom) actually exhibits two monolayers incoherently stacked, since twisted with a $6^{\circ}$ angle. (b) 2LG-AA, where all spot intensities on Ringl are equal and slightly higher than that on Ring2. (c) $2 \mathrm{LG}-\mathrm{AB}$, where the spot intensities on Ringl are distributed according to a three-fold symmetry, i.e. with an alternance of strong and weak spots, whereas all spot intensities on Ringl are lower than that on Ring2. Actual intensity values are provided in Table 1. Scale bars are $5 \mathrm{~nm}^{-1}$ 


\begin{tabular}{|c|c|c|c|c|c|c|c|c|c|c|c|}
\hline \multirow{2}{*}{$\begin{array}{l}\text { Stacking } \\
\text { sequence }\end{array}$} & \multirow{2}{*}{$\begin{array}{c}\left\|\overrightarrow{\mathbf{a}}_{\mathbf{1}}\right\| \\
{[\mathbf{\AA}]}\end{array}$} & \multicolumn{5}{|c|}{$5 \mathrm{keV}$} & \multicolumn{5}{|c|}{$100 \mathrm{keV}$ (but unstable under electron beam) } \\
\hline & & $\begin{array}{l}\text { Ring1 } \\
\text { weak }\end{array}$ & $\begin{array}{l}\text { Ring1 } \\
\text { strong }\end{array}$ & $\begin{array}{l}\text { Ring1(s)/ } \\
\text { Ring1(w) }\end{array}$ & Ring2 & $\begin{array}{c}\text { Ring2/ } \\
\text { Ring1(s) }\end{array}$ & $\begin{array}{l}\text { Ring1 } \\
\text { weak }\end{array}$ & $\begin{array}{l}\text { Ring1 } \\
\text { strong }\end{array}$ & $\begin{array}{l}\text { Ring1(s)/ } \\
\text { Ring1(w) }\end{array}$ & Ring2 & $\begin{array}{c}\text { Ring2/ } \\
\text { Ring1(s) } \\
\end{array}$ \\
\hline Graphane & 2.53 & $0.511 *$ & 0.714 & 1.4 & 0.539 & 0.8 & $0.590 *$ & 0.633 & 1.1 & 0.561 & 0.9 \\
\hline $\begin{array}{c}\text { Diamane } \\
\text { AA }\end{array}$ & 2.52 & 1.3 & 1.362 & 1 & 1.04 & 0.8 & $1.393 *$ & 1.393 & 1 & 1.24 & 0.9 \\
\hline $\begin{array}{c}\text { Diamane } \\
\text { AB }\end{array}$ & 2.53 & $0.056 *$ & 0.362 & 6.5 & 1.03 & 2.8 & 0.140 & $0.205^{*}$ & 1.5 & 1.228 & 6 \\
\hline
\end{tabular}

$*$ is along the $[10(0)]$ direction

Table 2 Identification chart for graphane and diamanes. Intensities reported are that of a single spot for each spot series (Ringl(weak), Ringl(strong), Ring2) for DPs obtained for an electron energy of 5 or 100 $\mathrm{keV}$, respectively. It is considered that an intensity ratio in the range $\sim 0.7-1.4$ cannot provide an intensity difference high enough for being visible to eye, and can be misled by intensity variations due to local deformations, specifically for a single layer material such as graphane (also see Table 1 caption) 

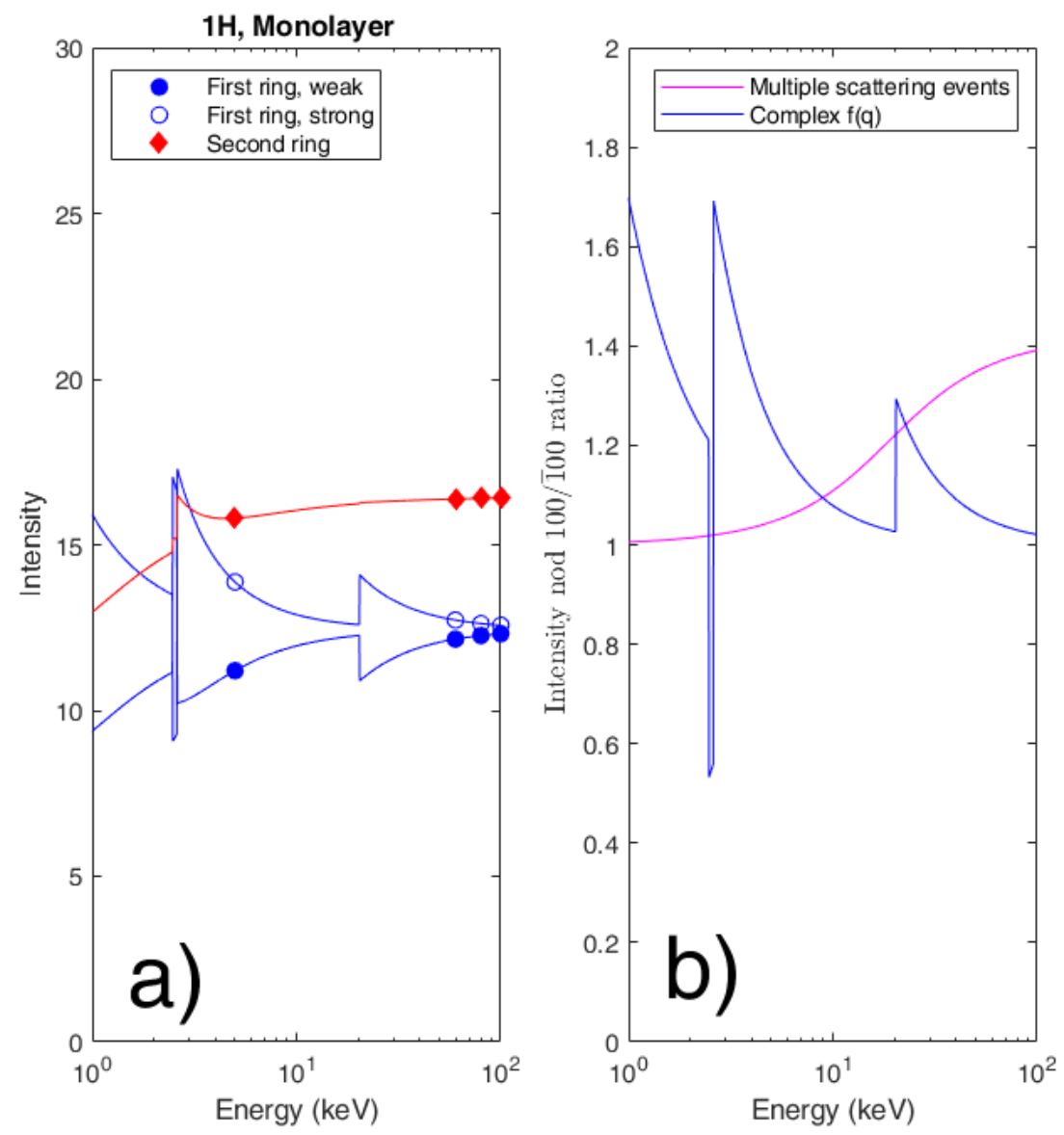

Fig. 3 (a) Variation of the calculated diffracted spot intensities while continuously changing the incident electron energy from 1 to $100 \mathrm{keV}$ for $\mathrm{MoS}_{2}$. Intensities are that for a single spot from each ring, Ring1 (blue line and circles) and Ring2 (red line and diamonds). Intensities specifically obtained for electron energies of 5,60,80, and $100 \mathrm{kV}$ TEM acceleration voltage are marked, for easier recognition. (b) Intensity ratio deduced from part (a) (blue line) and from multiple scattering events [11] 